\title{
Barriers to Clinical and Translational Research and Challenges of Investigator Initiated Multi-Center Clinical Trials
}

\author{
Laura Herbelin, BSc, CCRP - Research Instructor, Dept. of Neurology, \\ University of Kansas Medical Center \\ Richard Barohn, M.D., Chairman, Dept. of Neurology; Gertrude and Dewey \\ Ziegler Professor of Neurology; University Distinguished Professor; Vice \\ Chancellor for Research; President, Research Institute, University of Kansas \\ Medical Center
}

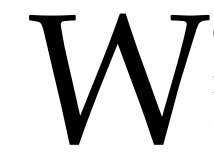

e have identified 10 barriers to overcome to conduct clinical translational research: 1) an idea, 2) interest/desire, 3) talent, 4) training, 5) time, 6) a team, 7) regulatory support, 8) space, 9) money and 10) subjects or study participants. We discuss the essential elements in overcoming these barriers, including programs initiated at KUMC for training and conducting clinical translational research. We also discuss challenges related to organizing and running a multicenter investigator initiated trial.

Spectrum of Translational Research

Translational research has now been divided into at least four categories. T1 research (Translation to Humans), involves pre-clinical animal studies, first in human safety, tolerability and pharmacokinetics studies. These first in human trials are conducted first in a normal population but often that is carried over into a population that the compound is targeting. The focus of this phase is discovery and safety. This corresponds to FDA Phase I trials.

The T2 research (Translation to $\mathrm{Pa}-$ tients) involves clinical trials that examine the effects of medication on a defined population. T2 research encompasses FDA categories of both Phase 2 and Phase 3 trials. FDA defines Phase 2 trial as a trial in which preliminary efficacy data with additional safety data is obtained. This usually this study involves less than 100 patients and depending on the population can be a single site or a multicenter trial. FDA Phase 3 trials are classified as a pivotal efficacy trial. These trials are large and often multicenter. The focus in T2 research is both on safety and efficacy.

T3 step is the Translation to Practice. The focus of T3 research is 'getting the word out' and putting that medication, procedure or behavioral intervention into practice, or implementation, dissemination and communication. In FDA terms, this is called post marketing Phase 4 research.

The next step is T4, Translation to Populations phase. These are usually community studies, policy studies and population outcome studies. The focus of this final phase is to improve population health. There is no FDA Phase equivalent 
to T4 research. Finally, T5 research has sometimes been used to describe international medical research.

Translational science refers to the study of methods in order to do translational research. This is a new concept that the NIH is now emphasizing. For example, comparing different types of recruitment tools to see which is most effective would be considered translational science.
6) a team, 7) regulatory support, 8) space,

9) money and 10) subjects or study participants.

Tools to overcome these barriers are available through multiple resources at medical centers, but the NIH/NCATS Clinical and Translational Science Award (CTSA) program is foremost in supplying these infrastructures to do CTR. At KU and in our Kansas City region our CTSA program is called Frontiers, the Heart-

\section{Spectrum of Translational Research}

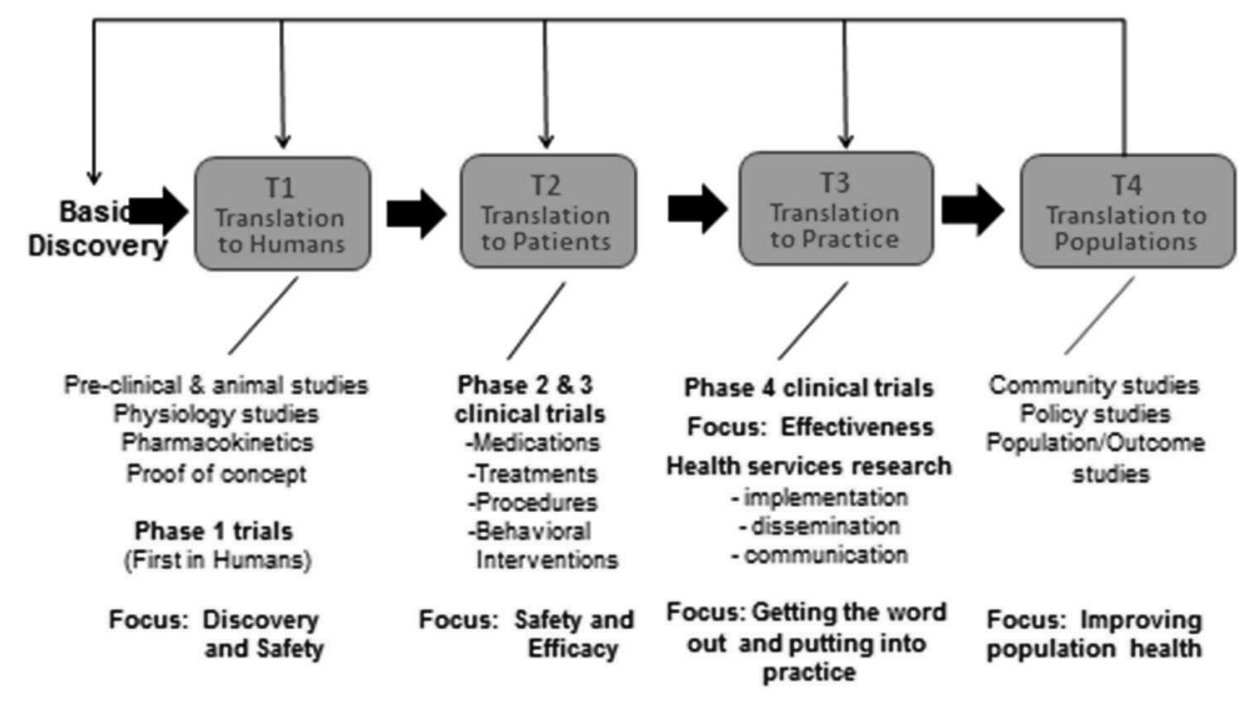

Figure 1: Spectrum of Translational Research

\section{Barriers (and Essentials) to Clinical and Translation Research \\ What barriers do investigators face} in attempting to conduct clinical/translational research? We have identified 10 barriers that investigators must overcome for a successful study. In essence there are also essential strategies because if you do not have them, you cannot overcome the barriers to initiate and conduct Clinical Translation Research (CTR). To do CTR, you must have 1) an idea, 2) interest/desire, 3) talent, 4) training, 5) time, land Institute for Clinical and Translational Research.

We successfully overcame these barriers in conducting several multi-center trials. We have recently coordinated and completed the following FDA Phase 2 (T2) studies:

- Therapeutic Trial of Mexiletine in NonDystrophic Myotonia FDA-IND \# 77,021(FDAOOPD - RO1-FD003454)1

- Safety and Tolerability Trial of Arimoclomol for Sporadic Inclusion Body Myositis FDA-IND \# 76,7732 
- Phase II Trial of Methotrexate in Myasthenia Gravis FDAIND \#101,306 (FDAOOPD - RO1-FD003538) ${ }^{3}$

- A Multi-Center Screening Trial of Safety and Efficacy of Rasagiline in Subjects with Amyotrophic Lateral Sclerosis (ALS) FDA-IND 104,3604

Only the mexiletine was a positive trial showing a definite benefit of the drug, for muscle stiffness in patients with rare genetic sodium or chloride mutation. Even in trials which do not have a "positive" result in favor of the drug, we learn a great deal of information regarding the CTR process by performing such studies. It is essential to publish results of negative or indeterminate trials so that health caregivers and patients can be informed. By law, it is now required to enter all data on a trial when it is complete and in clinicaltrials.gov. It is required to register all trials in clinicaltrials.gov at the start of a study and then to put the final data online.

We are currently coordinating the following multicenter trials:

- Phase 2 Study of Rasagiline for Treatment of Amyotrophic Lateral Sclerosis FDA IND\# 104,360 (FDAOOPD-R01FD003739) 5

- Patient Assisted Intervention for Neuropathy: Comparison of Treatment in Real Life Situations (PAIN-CONTRoLS) (CER-1306-02496) - a FDA Phase 4/T3 comparative effectiveness study. ${ }^{6}$

- Open Label study of subcutaneous immunoglobulin (SCIg) in myasthenia gravis FDA-IND \#: 15927 (Investigator Initiated - Pharmaceutical funded $)^{7}$

- Pilot Study of Acthar ${ }^{\circledR}$ Gel in Chronic Inflammatory Demyelinating Neuropa- thy FDA-IND \#126318 (Investigator Initiated - Pharmaceutical funded) - no clinicaltrials.gov assigned yet

- Phase II Study of Arimoclomol in IBM FDA-IND \# 76,773 (FDAOOPDRO1FD004809) - no clinicaltrials.gov assigned yet

The funding agencies for these trials have been the FDA Orphan Products Division (which funds rare disease research), the new Patient Center for Outcomes Research Institute (PCORI); and industry, through their investigator initiated grant programs. Let's examine the barriers mentioned above and how we overcame them.

\section{Barriers/Essentials to doing Multi-}

\section{Center Trials: Interest/Talent/}

Time/Training

Dr. Francis Collins, Director of the National Institutes of Health spoke at the Association for Clinical and Translational Science (ACTS) meeting in 2015. He reported that there was a crisis in the number of physicians doing research. How do you convince young faculty to include research in their career path and how do you train young physicians to conduct research?

The traditional $\mathrm{MD} / \mathrm{PhD}$ pathway usually does not lead medical students to CTR and clinical trials. Rather these students generally focus on early T1 laboratory research. We believe that $\mathrm{MD} / \mathrm{PhD}$ programs should be modified so that the PhD can be obtained in CTR. This is currently being done at a few medical schools but it may be the best pathway to get a study early in their career to pursue CTR post residency.

Currently in Frontiers, our NIH CTSA program, we do have a training 
program (TL1) where a medical student takes a year between their first and second year to do a CTR research project with a mentor and obtain a Master of Science in Clinical Research. Our TL1 program is open to Medical, Dental and Pharm D students but we have limited funds and train only four per year. To date we have had 16 students go through Frontiers TL1 program. Therefore, the number of students that can participate each year are few, but it does allow a selective number of students their first foray into CTR. The MS-CR program is 33 credit hours with the student thesis as the project.

Unfortunately, historically much of the training for CTR has been on the job and occurs during fellowship or junior faculty years for MDs/DOs. Through Frontiers, we have designed a core of 16 introductory lectures (Introduction to Clinical Research) that we encourage medical students, residents, fellows and PhD doctoral students to take. This is offered every Thursday night in a $1.5 \mathrm{hr}$ seminar each fall. For those interested in further education in CTR, they can take the courses that the TL- 1 students take over a longer time, 1 or 2 courses a semester. They can therefore obtain their MSCR over 2 to 5 years. This is often done by junior faculty.

There are some new CTR training activities at NIH/NCATS. One has come through a CTSA grant, Enhancing Clinical Research Professionals' Training and Qualifications (ECRPTQ), in which Tom Shanley, MD and Richard Barohn, MD are PIs. As a result of the ECRPTQ effort, it is now recommended that all investigators and coordinators involved in clinical trials complete and pass online Good Clinical Practice (GCP) training. This will become the new floor for training investigators and coordinators conducting trials. In the second phase of this initiative competency requirements for conducting clinical trials are being developed and additional training towards these competencies will be proposed. This process is expanding on an earlier joint task force on clinical trial competencies. ${ }^{8}$ In addition, the NCATS/CTSA Workforce Development Task Force (Richard Barohn cochair), is starting to develop these new courses for clinical trials research, and other training modules including one for community-engaged research.

As mentioned above, the majority of training that young investigators obtain is through on-the-job training. Usually the young faculty or student will have a senior clinical investigator to serve as a mentor and the training is accomplished through this mentorship. The following is an example from research project, Therapeutic Trial of Mexiletine in NonDystrophic Myotonia. Jeff Statland MD became involved with Dr. Barohn on this project immediately following medical school and throughout his neurology residency. This is unusual for a resident to be so actively involved in a multicenter clinical trial. Dr. Statland ended up becoming the first author of the paper published in JAMA. ${ }^{9}$ He then did a NIH funded Clinical Neurotherapeutics T32 Fellowship at the University of Rochester and he is now on faculty in the Department of Neurology at KUMC where he is designing and implementing neuromuscular trials. He is a KL2 scholar on the 
Frontiers CTSA NIH grant and he is mentoring studies, fellows, residents and junior faculty on CTR.

Additional barriers facing young investigators is finding time from clinical and teaching duties to participate in clinical research. There is a salary discrepancy between clinical income and income from research work. This is not a new problem. Dr. Francis Collins showed at the ACTS meeting in 2015 an article written by him in 1991 regarding titled Physician-Scientists: A Vanishing Breed in which he talks about the vanishing researcher and this income discrepancy. ${ }^{10}$

Dr. Christopher Austin, Director of NCATS, frequently emphasizes that we need a way to train and maintain careers of all researchers, whether they be full time principal investigators or team players who do mainly clinical work but serve as co-investigators on trials. Analogies can be made to other fields, such as the quarterback and linebackers in football, or the conductor and $2^{\text {nd }}$ violinist in a symphony, or the prima ballerina and sugar plum fairies in ballet. It is a challenge for both the leaders and essential team members to train and then remain involved in CTR.

Barriers/Essentials to doing MultiCenter Trials: Team Approach

'No man is an island, Entire of itself, Every man is a piece of the continent, A part of the main. If a clod be washed away by the sea, Europe is the less. As well as if a promontory were. As well as if a manor of thy friend's Or of thine own were: Any man's death diminishes me, Because I am involved in mankind, And therefore never send to know for whom the bell tolls; It tolls for thee'. - John Donne
A large team is required to conduct a clinical trial whether single site or multisite. Team members needed include but may not be limited to: principal investigator, co-investigators for each site (blinded and unblinded), project manager, study coordinators, clinical evaluators, biostatisticians, data manager/informatics, research pharmacy, laboratory/radiology personnel, budget/contracts, Internal Review Board (IRB), Data Safety Monitoring Board (DSMB)/Safety Monitors, patient advocacy groups, patients (to serve on study design and implementation committees) and external monitors. It is daunting to gather all of the team members, but just as daunting is coming up with the finances to pay for the team.

Barriers/Essentials to doing MultiCenter Trials: Sites on Team

Another barrier that is essential to overcome is determining which sites will be members of the study team. Many clinical trials require multiple sites, especially in rare disease research (the focus of our research), in which recruitment is a challenge due to the rarity of patients. Those sites chosen will become the sites in the trial where patients will be enrolled and placed on the study drug and followed throughout the study. As a leader of a multicenter trial, it is important to learn how to find and choose sites.

On a current study that we are recruiting for, Patient-Assisted Investigation of Neuropathic pain: Comparison of Treatments in Real-Life Situations, we need to utilize up to 40 sites to aid in recruiting 400 patients with painful neuropathy. Sites should be chosen based on if they have the patient population, and 
whether their research team is willing to recruit. We are often asked how a PI in Kansas can find sites to ask to be in a trial. This is not an easy question to answer but predominantly this is due to networking through academic societies where one becomes familiar with investigators over time. A site may get in a study and can demonstrate how well they perform in recruitment and regulatory efforts and if they do well, they will be asked back to future studies.

Another tool to aid in choosing sites is to become a member in a consortium. At KUMC, we currently are a member of the following consortia: Greater Plains Collaborative and the Vasculitis Patient Powered Research Network (both funded by PCORI), NeuroNEXT (funded by NIH), Alzheimer's Disease Cooperative Study (funded by NIA), Neurological Emergencies Treatment Trials and the NIH StrokeNet Nation Clinical Coordinating Center (both supported by NIH/NINDS). We are also a funded site of the National Cancer Institute. By becoming a member of a consortium, it allows you to gain a foothold in conducting clinical trials. Your experience will become a known commodity and more likely to be asked to participate in nonconsortium trials.

Barriers/Essentials to doing MultiCenter Trials: Regulatory

A major barrier that must be overcome is the handling of the regulatory issues faced in every study. Most of the issues are FDA regulatory issues. As the Principal Investigator (PI) at a site and the lead PI for the study, you are ultimately responsible for what occurs at each site. You must follow FDA guidelines. For instance, do you need to apply for an Investigational New Drug application or can you do the study under exempt status? The rules for this are available through the FDA. ${ }^{11}$ If you do apply for an IND, the FDA has to respond within 30 days. If they do not respond in 30 days, you can proceed with the study. If they do respond, you will most likely need to modify the protocol per their request. In most cases acknowledgement from the FDA is required before submission to the IRB is allowed. If you obtain a FDA IND, you have to file an annual updated with the FDA.

Another barrier that is essential to overcome is handling IRB approval from each site. Depending upon the study and the sites chosen to participate in the study, each site may have to submit to their own IRBs for approval. Recently there have been strides made in utilizing a single IRB on multicenter trials and there is a huge momentum at the NIH and PCORI levels to utilize a single IRB. ${ }^{12}$ There are several proposed solutions to this barrier. First is to utilize a Central IRB (cIRB). This has been utilized in industry for some time with commercial central IRBS but academic centralized IRBs are new. cIRBs generally focus on particular topic or disease (e.g., NeuroNext, NCI CIRB). A second option could be utilize a commercial IRB (e.g. Western IRB). These are often used for industry-sponsored multi-center trials. A third option is to utilize an IRB Share agreement. This is a joint review model and "Shared Review Process" in which a lead IRB approves a study; the Local Oversight IRB verifies agreement with the determination of the 
Lead IRB, and reviews local context issues. The fourth option is to utilize a Reliance model. This is a single IRB of record, chosen on a study-by-study basis, for the life of a study, involving a "reviewing IRB" and "relying institutions". The PCORnet Greater Plains Collaborative utilizes this model.

In a multicenter study the protocol must first be approved at the coordinating site, i.e. the IRB of record. Once it is approved, it is then sent to the other participating sites. The IRB of record will need to approve the consent forms from the participating sites prior to site activation. Once the participating sites are activated, they may begin enrolling study participants. The primary site acting as the IRB of record will track annual IRB approvals and track all adverse events and serious adverse events at all sites.

Barriers/Essentials to doing Multicenter trials: Monitoring

Pharmaceutical companies routinely do FDA compliant monitoring. This involves having a monitor periodically go through the research files on subjects enrolled in studies to ensure all proper research procedures are being followed. FDA released a White paper in 2007 titled The Food and Drug Administration's Oversight of Clinical Trials (OEI-0100160) which found that investigators conducting investigator initiated studies were deficient at monitoring their studies. ${ }^{13}$ There has recently been a push by the FDA for investigator-initiated studies to monitor their studies. Since then, the FDA released a draft guidance in 2013 that provides guidance for monitoring titled 'Oversight of Clinical InvestigationsA Risk-Based Approach to Monitoring'. ${ }^{14}$
The barriers to monitoring include supporting the infrastructure to carry out the monitoring, which includes personnel and the finances to hire them. Whereas pharmaceutical companies have the financial resources to carry out monitoring, investigator initiated studies using federal or other funds do not. The budgets for investigator initiated studies rarely have the capacity to fund a robust monitoring program. What we have done in our studies is a compromise so that we do remote monitoring and have sites send a selected number of study records for review. If they are deficient, a higher level of monitoring can be activated.

Barriers/Essentials to doing Multicenter trials: Recruitment

\section{Frontiers Registry}

No investigator initiated study will succeed if you cannot recruit the subjects needed for the study, making this a major barrier. At the University of Kansas Medical Center, we have arrived at some very innovative solutions. The Frontiers registry was developed out of our NIH CTSA program. All patients seen in a KUMC clinic are asked to sign up so they can potentially be contacted to be a research participant. As of January 2015, the Frontiers Registry contains nearly 40,000 potential research participants that were acquired from 17 different participating KUMC clinics. Use of the Registry has continued to grow and has been used by 49 different investigators (24 MDs and 25 PhDs) for 64 different protocols, a $50 \%$ increase over the last year. A variety of departments are using the registry spanning KU-Wichita, KU-Lawrence and UMKC. A total of 14,051 contacts have been provided to investigators. Of these, 
3423 were contacted by investigators resulting in 188 participants enrolled in studies. This experience was recently published by Dr. Kluding and colleagues from Frontiers. ${ }^{15}$

\section{Pioneers}

Another solution we have developed is the Pioneers Community Research Recruitment Registry, which also was developed in our NIH CTSA Frontiers program. The Pioneers Community Research Recruitment Registry was launched in September 2013. The Pioneers registry is an online, community-based research participant registry that can be used by investigators from multiple institutions within the Frontiers network. There are 2 primary objectives of the Pioneers Registry program: 1) to provide a universal portal for anyone in the community to "Become a Pioneer" and agree to be contacted for future research; 2) to provide an interactive website for listing studies that are actively recruiting participants.
Investigators may utilize the Pioneers program not only to advertise their study, and to request names and contact information for potential research participants using the results of a general medical history survey, but they may also include an interactive "I am interested" button in the description of their study. This allows potential participants to contact the study team directly, and may also include study-specific screening questions. These features were launched in Spring 2014 and are available to investigators to support their recruitment efforts.

\section{HERON}

The third solution is the Healthcare Enterprise Repository for Ontological Narration (HERON). HERON is a repository of de-identified clinical and biomedical data for clinical and translational research. HERON allows users to explore clinical data from multiple sources, housed in our EMR (Epic). Heron queries

Table 1: Complementary Recruitment Programs in Frontiers

\begin{tabular}{|l|l|l|}
\hline & Frontiers Registry & Pioneers Registry \\
\hline Status & $\begin{array}{l}\text { started in 2009; } \\
\text { approximately 40,000 } \\
\text { people }\end{array}$ & $\begin{array}{l}\text { started Sept 2013, currently 541 people } \\
\text { and 179 active studies listed }\end{array}$ \\
\hline Participant source & $\begin{array}{l}\text { U Kansas Physician } \\
\text { Clinic Patients only }\end{array}$ & Anyone \\
\hline Enrollment & $\begin{array}{l}\text { Via UKP Clinic } \\
\text { Registration; paper }\end{array}$ & Via website \\
\hline Link to EMR? & Yes & Optional \\
\hline Access to registrants & $\begin{array}{l}\text { KUMC researchers } \\
\text { only }\end{array}$ & $\begin{array}{l}\text { All Frontiers partners (e.g. KUMC, } \\
\text { Children's Mercy, St. Luke's, UMKC) }\end{array}$ \\
\hline $\begin{array}{l}\text { Ways to contact } \\
\text { registrants }\end{array}$ & $\begin{array}{l}\text { Phone or mail; email } \\
\text { for those signed up } \\
\text { since Sept 2014 }\end{array}$ & Email, phone, or mail \\
\hline
\end{tabular}


can provide a count of how many patients at KUMC meet the criteria of a clinical trial, and can provide additional patient observational data. HERON is linked to the Frontiers Research Participant Registry so that patients identified through HERON that participated in the registry can be contacted.

Barriers/Essentials to doing MultiCenter Trials: International Sites

Conducting clinical trials within the border of the United States can be challenging. Adding sites outside of our border adds an additional layer of complexity that few clinical investigators are aware of. For instance, if you have a site in Canada, you have to submit to Health Canada prior to the site initiating the trial. Health Canada is the Canadian version of the FDA IND. There are differences in terminology that we face with the application. Also, there is additional paperwork few US clinical investigators know about when transporting drug across the border for research. This information can be found at http://www.hcsc.gc.ca/dhp-mps/compli-conform/import-export/gui-0084_biu-uif-eng.php. ${ }^{16}$

Europe adds an additional barrier. There are European Union regulations that try to unify regulatory process for studies across Europe. However, each country often has their own additional requirements. One of the most expensive barriers to using a site in Europe is the need to hire a Qualified Person (QP). This person is hired to inspect the locations where the drug is processed. For instance in our mexiletine study, the QP not only had to inspect the drug manufacturing plant in Israel, but also had to inspect where the drug and placebo were over- encapsulated (University of Iowa's Research Pharmacy). There had to be separate contracts with the pharmacy, data coordinating center, as well as with the University of Kansas Medical Center. Medication had to be shipped from the University of Iowa's Research Pharmacy to a holding pharmacy where it had to be labeled. At that point, it could be shipped to the appropriate sites within Europe. Last but not least, customs at times would not release the drug pending a customs fee.

Using international sites is expensive for a budget on an investigator initiated trial and this needs to be factored into the budget. On the other hand, these international sites may be essential to meet enrollment needs for a study. We could not have completed our positive mexiletine in non-dystrophic myotonia study without the partnering of the Institute of Neurology in London, England, the University of Milan in Milan, Italy and University of Ontario in Canada.

\section{Conclusion}

There are significant barriers in carrying out a multicenter trial as the coordinating site. These barriers can be overcome but it takes personnel, infrastructure, time, training and money. Leading a multicenter study takes knowledge and skill and may not be for the timid or weak at heart. But the rewards are great and if you can overcome these many barriers discussed here, you and your team can have a significant impact in CTR and become leaders in your field. 
Acknowledgements: We would like to acknowledge funding by a Clinical and Translational Science Award from NCATS at NIH, UL1TR000001; a Clinical and Data Research Network award from PCORI as part of the PCORNet initiative, CDRN-130604631; a NeuroNEXT award from NINDS at NIH, U10NS077356; and a comparative effectiveness research project award from PCORI, CER-1306-02496.

\section{References:}

1. https://clinicaltrials.gov/ct2/show/NCT02336477

2. https://clinicaltrials.gov/ct2/show/NCT00769860

3. https://clinicaltrials.gov/ct2/show/NCT00814138

4. https://clinicaltrials.gov/ct2/show/NCT01232738

5. https://clinicaltrials.gov/ct2/show/NCT01786603

6. https://clinicaltrials.gov/ct2/show/NCT02260388

7. https://clinicaltrials.gov/ct2/show/NCT02100969

8. Sonstein, S.A., Seltzer, J., Li, R., Silva, H., Jones, C.T., Daemen, E. Moving from compliance to competency: A harmonized core competency framework for the clinical research professional. Clinical Researcher. 2014. 28; 17-23.

9. Statland JM, Bundy BN, Wang Y, Rayan DR, Trivedi JR, Sansone VA, Salajegheh MK, Venance SL, Ciafaloni E, Matthews E, Meola G, Herbelin L, Griggs RC, Barohn RJ, Hanna MG, and the Consortium for Clinical Investigaton of Neurologic Channelopathies. Mexiletine for symptoms and signs of myotonia in nondystrophic myotonia: a randomized controlled trial. JAMA 2012; 308:1357-1365. PMCID: PMC3564227. NIHMSID: NIHMS429387

10. Collins, F.S. Physician scientists: A vanishing breed, Yale Medicine, 1991-1992: 26: 5-8.

11. Website: http://www.fda.gov/Drugs/DevelopmentApprovalProcess/HowDrugsareDevelopedandApproved/ApprovalApplications/InvestigationalNewDrugINDApplication/

12. Website: http://www.hhs.gov/ohrp/humansubjects/regulations/nprm2015summary.html

13. Website: http://oig.hhs.gov/oei/re-ports/ oei-01-06-00160.

14. Website: http://www.fda.gov/downloads/Drugs/.../Guidances/UCM269919.pdf

15. Kluding PM, Denton J, Jamison TR, Brooks W, Blackwell K, Lantos JD, Waitman LR, McMahon MB, Choudhary A, Bott MJ, Greiner A, Klaus S, O'BrienLadner A, Aaronson LS, Burns J, Barohn R. Frontiers: Integration of a Research Participant Registry with Medical Clinic Registration and Electronic Health Records. Clin Transl Sci.2015; 3 Aug. http:// onlinelibrary.wiley.com/doi/10.1111/cts.12309/a bstract?campaign=wolearlyview).

16. Website: http://www.hc-sc.gc.ca/dhpmps/compli-conform/import-export/ gui-0084_biu-uif-eng.php. 